\title{
Treemaps for Directed Acyclic Graphs ${ }^{\star}$
}

\author{
Vassilis Tsiaras, Sofia Triantafilou, and Ioannis G. Tollis \\ Institute of Computer Science, Foundation for Research and Technology-Hellas, \\ Vassilika Vouton, P.O. Box 1385, Heraklion, GR-71110 Greece \\ \{tsiaras, striant,tollis\}@ics.forth.gr \\ http://www.ics.forth.gr \\ and \\ Department of Computer Science, University of Crete, \\ P.O. Box 2208, Heraklion, Crete, GR-71409 Greece \\ \{tsiaras, striant, tollis\}@csd.uoc.gr \\ http://www.csd.uoc.gr
}

\begin{abstract}
Gene Ontology information related to the biological role of genes is organized in a hierarchical manner that can be represented by a directed acyclic graph (DAG). Treemaps graphically represent hierarchical information via a two-dimensional rectangular map. They efficiently display large trees in limited screen space. Treemaps have been used to visualize the Gene Ontology by first transforming the DAG into a tree. However this transformation has several undesirable effects such as producing trees with a large number of nodes and scattering the rectangles associated with the duplicates of a node around the screen. In this paper we introduce the problem of visualizing a DAG as a treemap, we present two special cases, and we discuss complexity results.
\end{abstract}

Keywords: Treemap, Directed Acyclic Graph (DAG) Visualization, Gene Ontology.

\section{Introduction}

The Gene Ontology Consortium (GO) 14 databases store thousands of terms that describe information related to the biological role of genes. The information in GO is organized in a hierarchical manner where the terms are placed in layers that go from general to specific. The GO organization can be represented by a directed acyclic graph (DAG) where the set of vertices $V$ is the set of terms and an edge is used to declare the $i s_{-} a$ or part_of relationship between two terms.

Treemaps graphically represent hierarchical information via a two-dimensional rectangular map, providing compact visual representations of large trees through area, color and shading effects 3511. Treemaps, have also been used to visualize compound graphs that contain both hierarchical relations and adjacency relations [7].

* This work was supported in part by INFOBIOMED code: IST-2002-507585 and the Greek General Secretariat for Research and Technology under Program "ARISTEIA", Code 1308/B1/3.3.1/317/12.04.2002. 
In the context of GO, treemaps have been used to visualize microarray data, where each gene transcript is assigned all possible paths that start from it and terminate to the root (the "all" term) of GO 1. Symeonidis et al. in 12 proposed to decompose the complete GO DAG into a tree by duplicating the nodes with many in-coming edges, and then to use a treemap algorithm to visualize the tree, see Figure 1. The duplication of a node however triggers the duplication of all of its descendants. Therefore the transformation of a DAG into a tree leads to trees with (potentially exponentially) many more nodes than the original DAG. Symeonidis et al. in 12 reported that the initial GO DAG had $\sim 20.000$ terms, while the produced equivalent tree had $\sim 100.000$ terms. Another drawback of duplicating the nodes is that the rectangles associated with the multiple replicas of a node are scattered around the screen. In this paper we introduce the

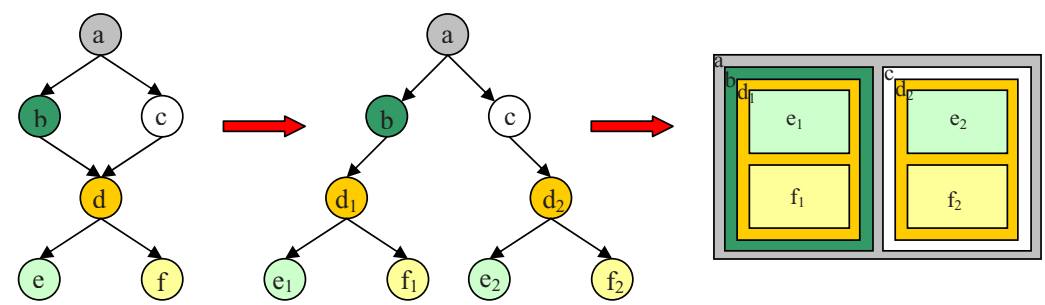

Fig. 1. Example of transforming a DAG into a tree and then drawing it as a treemap

problem of drawing a DAG as a treemap without converting it to a tree first. We consider several variations of the problem, we present some characterizations of simple families of DAGs that admit such a drawing, and provide complexity results for the general problem.

\section{Problem Definition}

\subsection{Notations}

Suppose that $G=(V, E)$ is a layered directed acyclic graph (DAG) with a partition of the node set $V$ into subsets $L_{1}, L_{2}, \ldots, L_{h}$, such that if $(u, v) \in E$, where $u \in L_{i}$ and $v \in L_{j}$, then $i>j$. Without loss of generality we assume that the layering is proper, since the "long" edges that span more than two layers may be replaced by paths having dummy vertices in the internal layers [2].

Let $R_{v}$ denote the display region of a node $v \in V$. Every directed edge $e=$ $(u, v)$ from a node $u$ to a node $v$ corresponds to a drawing region $R_{e}=R_{v} \cap R_{u}$ which is the part of the child's drawing region $R_{v}$ that is drawn within the parent's drawing region $R_{u}$.

Given a vertex $v \in V$ we denote the set of its in-coming and out-going edges by $\Gamma^{-}(v)=\{e \in E /$ destination $(e)=v\}$ and $\Gamma^{+}(v)=\{e \in E / \operatorname{origin}(e)=v\}$ respectively. 


\subsection{Treemap Drawing Constraints}

Treemaps have the invariant that the drawing rectangle of any node (different from the root) is contained within the drawing rectangle of its parent. When the graph is a tree this invariant can easily be satisfied since every node has one parent. When the graph is a DAG, the above invariant should be replaced by the invariant that the drawing rectangle of any node is contained within the union of the rectangles of its parent nodes. Apart from this invariant it is plausible to assume that the drawing rectangles of sibling nodes do not overlap and that the drawing rectangle of a parent node is covered by the drawing rectangles of its children nodes. The above invariant and assumptions are summarized in the following definition.

Definition 1 (Treemap basic drawing constraints). The drawing is constrained by the following rules.

B1. The display area of the DAG (screen) is a rectangle.

B2. Every node is drawn as a rectangle $\left(R_{v}\right.$ is a rectangle for every $\left.v \in V\right)$.

B3. If two distinct nodes $u, v \in V$ are assigned to the same layer their rectangles do not overlap (area $\left.\left(R_{u} \cap R_{v}\right)=0\right)$.

B4. The rectangle of a child node occupies a non-zero area in each one of its parent node rectangles. $\left(\right.$ area $\left(R_{e}\right)=\operatorname{area}\left(R_{u} \cap R_{v}\right) \neq 0$ if $\left.e=(u, v) \in E\right)$.

B5. The rectangle of a child node is contained in the union of rectangles of its parent nodes $\left(R_{v} \subset \cup_{(u, v) \in E} R_{u}\right)$.

$B 6$. The rectangle of a parent node is covered by the rectangles of its children nodes $\left(R_{u} \subset \cup_{(u, v) \in E} R_{v}\right)$.

The drawing rules of the above definition are quite general since they do not constrain the area of the leaf nodes, and the proportion of a child's node area that is drawn on each one of its parent rectangles. To simplify the analysis of the problem we constraint these two parameters by making the following assumptions.

\section{Definition 2 (Treemap additional drawing constraints)}

A1. The leaf nodes are drawn in equal area ( $\left.\frac{\text { screen area }}{\text { number of leaf nodes }}\right)$ rectangles.

A2. The drawing rectangle of a child node occupies the same area on each one of its parent rectangles (For every non source node $v$, area $\left(R_{e}\right)=\frac{\operatorname{area}\left(R_{v}\right)}{\left|\Gamma^{-}(v)\right|}$, for every $\left.e \in \Gamma^{-}(v)\right)$.

In the following we will use the term treemap drawing to characterize a drawing according to the basic and additional drawing constraints.

Having defined the drawing rules, we can define the following problems:

1. Given a DAG $G_{1}$, does $G_{1}$ admit a treemap drawing?

2. In case that the answer to the first problem is negative, what is the minimum number of node duplications that are needed to transform $G_{1}$ into a DAG $G_{2}$ that admits a treemap drawing? 


\subsection{Examples and Counter-Examples of DAGs That Admit a Treemap Drawing}

Examples of DAGs that admit a treemap drawing appear in Figure2, From the

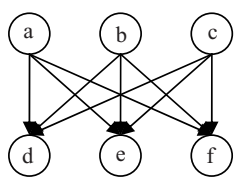

(a) The $K_{33}$ DAG
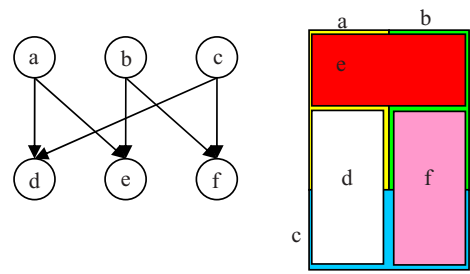

(b) A "circular" DAG

Fig. 2. Examples of DAGs that can be drawn as treemaps

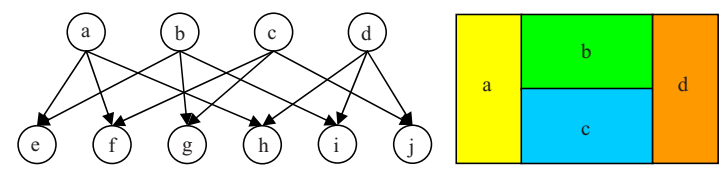

(a) Consecutive conditions violation

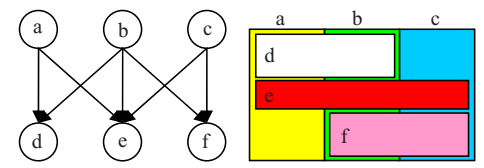

(b) Area imbalance

Fig. 3. Examples of DAGs that do not admit a treemap drawing

counter-examples of Figure 3 we see that there are DAGs that cannot be drawn as treemaps. The DAG in Figure 3(a) cannot be drawn due to adjacency constraint violation. The leaf nodes $e, f, g, h, i, j$ constrain the parent nodes $a, b, c, d$ to be drawn in adjacent rectangles. However we cannot have a configuration where all the pairs $\{a, b\},\{a, c\},\{a, d\},\{b, c\},\{b, d\},\{c, d\}$ of rectangles are adjacent. In this case in order to draw the DAG we can either duplicate one of the nodes $e, f, g, h, i, j$ or draw one of these nodes using two disjoint rectangles. The two operations are similar and when applied to child node remove the corresponding adjacency constraint.

In general, due to the four color (map coloring) theorem there exists a counterexample involving five parent nodes and ten children nodes (one child node for every pair of parent nodes), even in the case that we relax constraint B2, allowing drawings to be simply connected regions of the plane.

The example of Figure 3(b) shows a DAG that does not admit a treemap drawing, due to area imbalance among the first layer nodes $a, b, c$. Assuming 
that the leaf nodes have unit area, then nodes $a$ and $c$ have area $1 / 2+1 / 3$ while node $b$ has area $1+1 / 3$. However, if we relax constraint A2, then this DAG admits a treemap drawing.

\subsection{Node Duplication}

Usually, a DAG encountered in practice does not admit a treemap drawing. In this case we should relax one or more of constraints B1-B6, A1-A2 or change the form of the DAG. Symeonidis et al. in 12 chose to transform the DAG into a forest of trees by multiple node duplications. An example of a node duplication is shown in Figure 3(b) where after the creation of two replicas of node $e$, one with two parents and one with one parent, the DAG is transformed into a new DAG which admits a drawing, see Figure 4

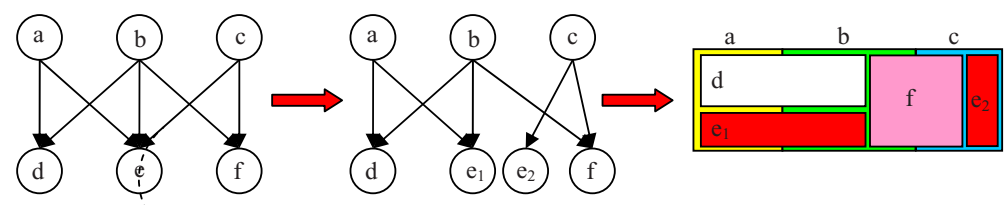

Fig. 4. After the duplication of node $e$ the DAG of Figure 3 is transformed into a DAG that has a treemap drawing

\section{Special Cases}

We will continue by considering two special cases. The first case is based on a restricted form of DAGs, the second on a restricted form of treemaps.

\subsection{Two Terminal Series Parallel Digraphs}

A Two Terminal Series Parallel (TTSP) digraph is recursively defined as follows 213. An edge joining two vertices is a TTSP digraph. Let $G_{1}$ and $G_{2}$ be two TTSP digraphs. Their series and parallel compositions, defined below, are also TTSP digraphs.

- The series composition of $G_{1}$ and $G_{2}$ is the digraph obtained by identifying the sink of $G_{1}$ with the source of $G_{2}$.

- The parallel composition of $G_{1}$ and $G_{2}$ is the digraph obtained by identifying the source of $G_{1}$ with the source of $G_{2}$ and the sink of $G_{1}$ with the sink of $G_{2}$.

Due to its recursive structure a TTSP digraph always admits a treemap drawing. The base TTSP digraph is drawn as a rectangle. In a series composition the rectangle of graph $G_{2}$ is drawn on the top of the rectangle of graph $G_{1}$. In a parallel composition the rectangle of the composite graph is sliced into the rectangles of $G_{1}$ and $G_{2}$. 


\section{Algorithm}

1. Construct the decomposition tree of $G[13$ and merge the adjacent $P$-nodes. In the resulting tree the $P$-nodes may have two or more children.

2. Using the decomposition tree calculate the size of the components.

(a) In a series composition $\operatorname{size}(G)=\operatorname{size}\left(G_{1}\right)=\operatorname{size}\left(G_{2}\right)$.

(b) In a parallel composition size $(G)=\operatorname{size}\left(G_{1}\right)+\operatorname{size}\left(G_{2}\right)+\ldots+\operatorname{size}\left(G_{k}\right)$.

3 . Using the decomposition tree recursively draw the component rectangles.

(a) The rectangles have area proportional to the size of the corresponding component.

(b) In a series composition, the rectangles of the two components coincide.

(c) In a parallel composition, use any of the existing treemap algorithms to lay out the component rectangles.

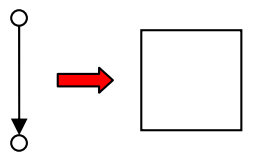

(a) The base TTSP digraph

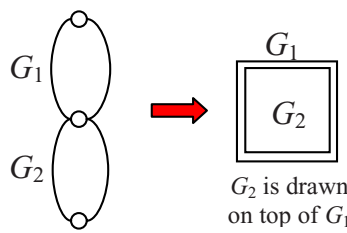

(b) Series composition

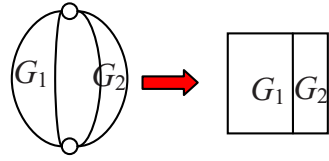

(c) Parallel composition

Fig. 5. Recursive definition of a TTSP digraph and the corresponding recursive treemap drawings

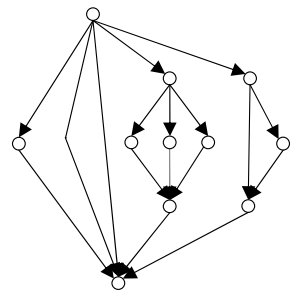

(a) A TTSP

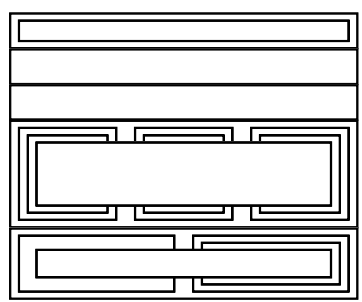

(b) Slice and Dice layout

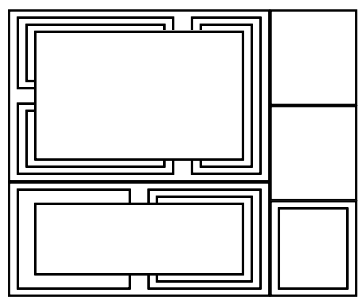

(c) Squarified layout

Fig. 6. Example of a TTSP digraph treemap drawing

\subsection{One Dimensional Treemaps}

Definition 3. A treemap is called one dimensional if the rectangle representing a node is divided with vertical (or horizontal) lines into smaller rectangles representing its children and the orientation of the lines is the same for all the nodes of a hierarchy. 
Since the height (resp. width) of all the rectangles is constant and equal to the height (resp. width) of the screen, the problem is one dimensional and the rectangles $R_{q}$ can be represented by intervals $I_{q}$. Also only the ordering and not length of the intervals $I_{e}=I_{u} \cap I_{v}, e=(u, v) \in E$ constrain the problem. For this reason we consider the out-going edges of every non-leaf node $u \in V$ as subnodes inside the node. The in-coming edges of a node can be considered as in-coming edges of every one of its subnodes. A drawing $\cup_{n_{e} \in L_{k}} I_{n_{e}}$ of the subnodes $n_{e} \in L_{k}$ of layer $k \in\{2, \ldots, h\}$ corresponds to an ordering of the subnodes $n_{e} \in L_{k}$.

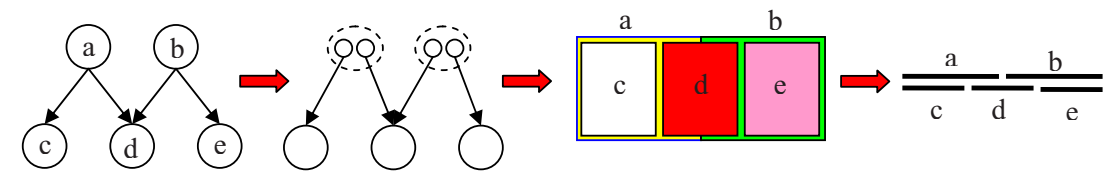

Fig. 7. A one dimensional treemap example. A subnode is created for each out-going edge.

Definition 4 (ONE DIMENSIONAL TREEMAP FOR DAG). The recognition problem

INSTANCE: A DAGG.

QUESTION: Can $G$ be drawn as a one dimensional treemap?

Suppose that $u$ is a node in layer $L_{k}$. We will give the necessary and sufficient conditions that the ancestor subnodes of $u$ must satisfy in order to be able to draw $I_{u}$ as an interval. With the term ancestor subnodes we mean the subnodes reachable from node $u$ if the direction of the edges is reversed.

Let $P_{u, i}$ denote the set of ancestor subnodes of node $u \in L_{k}$ in layer $i \in$ $\{k+1, \ldots, h\}$.

Theorem 1 (Necessary conditions). Suppose that in a one dimensional treemap drawing of a graph $G=(V, E)$ a node $u \in L_{k}$ can be drawn as an interval $I_{u}$. Then the union of the drawings of the ancestor subnodes of $u$ in layer $i, \cup_{e \in P_{u, i}} I_{e}$, is an interval for every layer $i \in\{k+1, \ldots, h\}$.

Proof. By induction on the layers $L_{k+j}, j=0, \ldots, h-k$.

For $j=0, I_{u}$ is an interval by the hypothesis.

Now, suppose that for $j=0, \ldots, i<h-k$, there is an ordering of the subnodes in every one of the layers $k+j, \ldots h$, such that $\cup_{e \in P_{u, k+j}} I_{e}$ to be an interval, but $\cup_{e \in P_{u, k+j+1}} I_{e}$ cannot be an interval. Then there is at least one node $v \in L_{k+j+1}, v \notin P_{u, k+j+1}$, which is between two nodes $\alpha, \beta \in P_{u, k+j+1}$. Then the interval $\cup_{e \in P_{u, k+j}} I_{e}$ intersects the intervals $I_{\alpha}$ and $I_{\beta}$ but not the interval $I_{v}$, a contradiction.

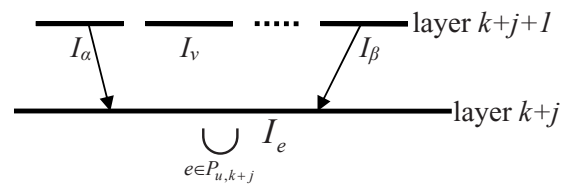


Theorem 2 (Sufficient condition). If there is an ordering of the subnodes in $L_{k+1}$ such that the parent subnodes $P_{u, k+1}$, of a node $u \in L_{k}, k<h$ are consecutive in this ordering then $u$ can be drawn as an interval.

Proof. Since $P_{u, k+1}$ are consecutive $\cup_{e \in P_{u, k+1}} I_{e}$ is an interval. Then simply draw $I_{u}$ in this interval.

\section{Algorithm}

From the above theorems every non-source node $u \in L_{k}$ defines constraints on the admissible subnode permutations in each one of the layers $L_{i}, i \in\{k, \ldots, h\}$. Therefore the decision problem is transformed to $h-1$ consecutive ones decision problems 49]. One consecutive ones problem for each layer $L_{i}, i \in\{2, \ldots, h\}$.

There is one list list $_{i}$ of constraints for each layer $L_{i}, i \in\{2, \ldots, h\}$. Initially the lists are empty. Then we add the constraints as follows.

for $i=2$ to $h$ do

for $v \in L_{i}$ do

add to the $l_{i s t_{i}}$ the constraint that the subnodes of $v$ are consecutive.

for $i=1$ to $h-1 \mathrm{do}$

for $v \in L_{i}$ do

for $j=i+1$ to $h$ do

add to the $l i s t_{j}$ the constraint that the subnodes $P_{v, j}$ are consecutive.

\section{Complexity analysis}

Without loss of generality we assume that there are no leaf nodes in layers $2, \ldots, h$.

Suppose that $n_{i}=\left|L_{i}\right|, i \in\{1, \ldots, h\}$ and that $m_{i}$ edges go from layer $i$ to layer $i-1, i \in\{2, \ldots, h\}$.

For $i \in\{2, \ldots, h\}$ the list list $_{i}$ has at most $n_{i}$ trivial constraints and at most $n_{j}$ constraints due to nodes at layer $L_{j}, j<i$. In total it has $n_{i}+\ldots+n_{1}$ constraints. Each costraint has size at most $m_{i}$.

Therefore the total time is:

$$
\sum_{i=2}^{h} O\left(m_{i} \cdot\left(n_{i}+\ldots+n_{1}\right)\right)=\sum_{i=2}^{h} O\left(m_{i} \cdot n\right)=O(m \cdot n)
$$

which is polynomial on the input size $m=\sum_{i=1}^{L} m_{i}$ and $n=\sum_{i=1}^{L} n_{i}$

\section{The General Case}

\subsection{The Recognition Problem}

Taking the nodes of a layer $L_{k}$ isolated from the rest of the DAG, the problem is similar to a floorplan problem where the display area is dissected into $n_{k}=\left|L_{k}\right|$ 
soft rectangles, i.e., rectangles whose area is fixed but their dimensions may vary. The number of possible dissections (the solutions space) is bounded below by $\Omega\left(n_{k} ! 2^{3 n} / n_{k}^{4}\right)$ and above by $O\left(n_{k} ! 2^{5 n} / n_{k}^{4.5}\right)$ [10].

Considering two consecutive layers $L_{k+1}$ and $L_{k}$ of a DAG, the layouts of the two layers are constrained by the edges among the two layers, according to the drawing rules. The combined solution space may be empty or contain a number of solutions. We will show that deciding whether the solution space is empty or not is NP-complete.

Definition 5 (TREEMAP FOR DAG). The recognition problem INSTANCE: A DAGG.

QUESTION: Can $G$ be drawn as a treemap?

Theorem 3. The TREEMAP FOR DAG decision problem is NP-complete even if we restrict it to two layer weakly connected DAGs.

Proof. Given a dissection of the display area (screen) into $\left|L_{k}\right|$ rectangles for each layer $L_{k}, k \in\{1, \ldots, h\}$ of a DAG $G$, we can check in polynomial time if these dissections correspond to a treemap drawing of $G$. Therefore the problem belongs to NP.

Next we will show that the problem TREEMAP FOR DAG is NP-hard. The proof will be done by reducing the 3-PARTITION problem to a restricted version of the TREEMAP FOR DAG problem. Namely, as input we consider only two layer DAGs. For simplicity of the proof we will allow an input DAG to be composed of several weakly connected components.

\section{Definition 6 (3-PARTITION).}

INSTANCE: A multiset $A$ of $3 m$ positive integers $A=\left\{\alpha_{1}, \alpha_{2}, \ldots, \alpha_{3 m}\right\}$ where the $\alpha_{i}$ 's are bounded above by a polynomial in $m$ and $\frac{\Sigma}{4}<\alpha_{i}<\frac{\Sigma}{2}$, where $\Sigma=\frac{1}{m}\left(\alpha_{1}+\alpha_{2}+\ldots+\alpha_{3 m}\right)$.

QUESTION: Can $A$ be partitioned into $m$ triples $A_{1}, A_{2}, \ldots, A_{m}$ such that each triple has the same sum. Specifically each triple must sum to $\Sigma$.

The condition $\frac{\Sigma}{4}<\alpha_{i}<\frac{\Sigma}{2}$ forces every set of $\alpha_{i}$ 's summing to $\Sigma$, to have size exactly 3 . The 3 -PARTITION is strongly NP-complete since it remains NPcomplete even when representing the numbers in the input instance in unary [6]. The reduction is done by local replacement and using an enforcer.

Enforcer: The DAG used as enforcer has $2 m+2$ nodes at the second layer. The nodes $\beta$ and $1,2, \ldots, 2 m+1$. At the first layer there are $(m+1) \Sigma+6 m+4$ nodes. Each of the nodes $1^{\prime}, 2^{\prime}, \ldots,(2 m+1)^{\prime}$ has two parents. One is node $\beta$ and the other is the corresponding numbered node in the first layer. The $\beta$-node rectangle is drawn in one side of the enforcer and precludes any other rectangle to be drawn along this side. Also the $\beta$-node together with the $\gamma$-node force the nodes $1,2, \ldots, 2 m+1$ to be drawn as consecutive rectangles. For every pair of $(j, j+1)$ of second layer nodes there exists a first layer node which has them as parents and constrains them to be consecutive. The second node which has as parents the nodes $j$ and $j+1$ is used for completing the drawing (garbage 


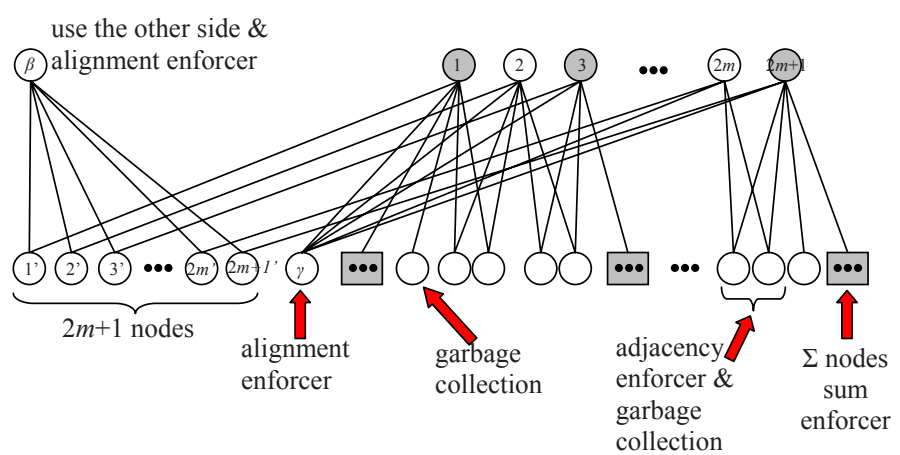

Fig. 8. The enforcer used in the proof
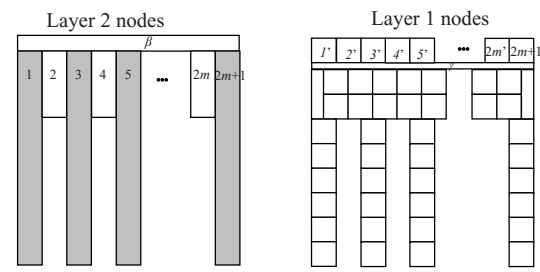

Fig. 9. One possible drawing of the enforcer. The rectangles $1,2, \ldots, 2 m+1$ are forced by the rectangle $\gamma$ to have the same width.

collection). Also for garbage collection one node is connected to node 1 and one node to node $2 m+1$.

Finally, every odd numbered node of the second layer has $\Sigma$ children nodes.

Local replacement: For each $\alpha_{i} \in A$ we consider a two layer DAG which has one node at layer two (parent node) and $\alpha_{i}$ nodes at layer one (children nodes).

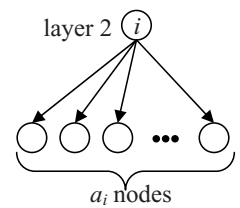

layer 2
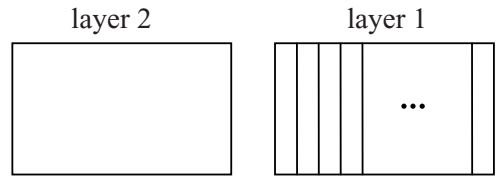

The drawing of node $i$ is a rectangle with area $\alpha_{i}$, but without any constraint on the aspect ratio of its sides. In order for the final drawing to be a rectangle the $3 m$ rectangles should fill the holes of the enforcer.

The reduction from the 3-PARTITION to TREEMAP FOR DAG uses polynomial number of resources since the numbers involved in 3-PARTITION are bounded by a polynomial in $m$.

The 3-PARTITION instance has a solution if and only the TREEMAP FOR DAG instance has a solution since in every hole can fit exactly three rectangles. 


\subsection{Minimization of Node Duplication}

\section{Definition 7 (MINIMUM DUPLICATION OF NODES).}

INSTANCE: $A D A G G_{1}$ and an integer $K$.

QUESTION: Can $G_{1}$ be transformed into a DAG $G_{2}$ that admits a treemap drawing by duplicating at most $K$ nodes.

Comment: The problem MINIMUM DUPLICATION OF NODES is NPcomplete since its restriction for $K=0$ is the problem TREEMAP FOR DAG, which is NP-complete.

\section{Discussion}

In this paper we introduced the problem of drawing a DAG as a treemap. We defined the recognition and minimization problems and we showed that in the general case they are NP-complete and NP-hard respectively. We also considered two special cases by restricting the form of the DAG and of the treemap respectively. We are currently investigating drawing heuristics based on relaxations of the drawing constraints and $\backslash$ or restrictions on the form of DAGs. The results of this research will be published in a subsequent paper concerning the application of these techniques to hierarchically organized ontologies.

\section{References}

1. Baehrecke, E., Dang, N., Babaria, K., Shneiderman, B.: Visualization and analysis of microarray and gene ontology data with treemaps. BMC Bioinformatics 5(84) (2004)

2. Di Battista, G., Eades, P., Tamassia, R., Tollis, I.G.: Graph Drawing: Algorithms for the Visualization of graphs. Prentice - Hall, New Jersey, U.S.A. (1998)

3. Bederson, B., Shneiderman, B., Wattenberg, M.: Ordered and quantum treemaps: Making effective use of $2 \mathrm{D}$ space to display hierarchies. ACM Transactions on Graphics 21(4), 833 (2002)

4. Booth, S., Lueker, S.: Testing for the consecutive ones property, interval graphs, and graph planarity using PQ-tree algorithms. Journal of Computer and System Sciences 13, 335 (1976)

5. Bruls, M., Huizing, K., van Wijk, J.J.: Squarified treemaps. In: Proceedings of Joint Eurographics and IEEE TCVG Symposium on Visualization, p. 33. Springer, Heidelberg (2000)

6. Garey, M., Johnson, D.: Complexity results for multiprocessor scheduling under resource constraints. SIAM Journal on Computing 4(4), 397 (1975)

7. Holten, D.: Hierarchical Edge Bundles: Visualization of Adjacency Relations in Hierarchical Data. IEEE Transactions on Visualization and Computer Graphics 12(5), 741 (2006)

8. Hsu, W.-L.: PC-Trees vs. PQ-Trees. In: Wang, J. (ed.) COCOON 2001. LNCS, vol. 2108, p. 207. Springer, Heidelberg (2001)

9. Meidanis, J., Porto, O., Telles, G.: On the consecutive ones property. Discrete Applied Mathematics 88, 325 (1998) 
10. Shen, Z.C., Chu, C.: Bounds on the Number of Slicing, Mosaic, and General Floorplans. IEEE Transactions on Computer-Aided Design of Integrated Circuits and Systems 22(10), 1354 (2003)

11. Shneiderman, B.: Tree visualization with tree-maps: 2-d space-filling approach. ACM Transactions on Graphics 11(1), 92 (1992)

12. Symeonidis, A., Tollis, I., Reczko, M.: Visualization of Functional Aspects of microRNA Regulatory Networks Using the Gene Ontology. In: Maglaveras, N., Chouvarda, I., Koutkias, V., Brause, R. (eds.) ISBMDA 2006. LNCS (LNBI), vol. 4345, pp. 13-24. Springer, Heidelberg (2006)

13. Valdes, J., Tarjan, R., Lawler, E.L.: The recognition of Series Parallel digraphs. SIAM Journal on Computing 11, 289-313 (1982)

14. http://www.geneontology.org 